\title{
ARQUITECTURA Y ORNAMENTO: SUBJETIVIDAD ORIENTADA A LOS OBJETOS Y POLÍTICA DEL POST-TRABAJO
}

DOI: https://doi.org/10.18861/ania.2019.9.2902

Arq. Mariana Righi M.Sc.

http://orcid.org/0000-0002-8022-5909 


\section{ARQ. MARIANA RIGHI M.SC.}

Master of Science in Design with concentration in Advance Architectural Design, Universidad de Pennsylvania (Estados Unidos). Arquitecta, Universidad de Buenos Aires (Argentina). Investigadora y docente, Autonomous Manufacturing Lab, Universidad de Pennsylvania (Estados Unidos).

FECHA DE RECEPCIÓN: 12 de mayo de 2019.

FECHA DE ACEPTACIÓN: 21 de mayo de 2019.

REGISTRO BIBLIOGRÁFICO: RIGHI, M. (2019). Arquitectura y ornamento: subjetividad orientada a los objetos y política del post-trabajo. Anales de Investigación en Arquitectura, 9 (1), 65-82. 


\section{RESUMEN}

Según Antonie Picon en Ornament: the politics of architecture and subjectivity, el retorno de la ornamentación como práctica en la arquitectura contemporánea tiene una serie de continuidades con el ornamento clásico en términos de subjetividad y política.

En este contexto, el primer objetivo de este artículo es revisar la idea de subjetividad en la práctica ornamental/ arquitectónica contemporánea desde el punto de vista de la relación "object-oriented". Tomando un objeto creado por un sistema de multi-agentes y fabricado con un extrusor de arcilla adjunto a un brazo robótico, nos preguntaremos en que consiste la práctica ornamental contemporánea si la existencia del sujeto ya no es determinante y regulador de su diseño y ejecución.

En un segundo lugar, el objetivo es especular con la posibilidad de que, en el contexto del post-trabajo y la plena automatización de la producción, los diseñadores reencuentren el significado de las formas arquitectónicas que doten de un sentido político al ornamento de hoy.

Palabras clave: Ornamento, arquitectura, post-humanismo, post-trabajo, programación en base a agentes, programación orientada a objetos, fabricación digital, fabricación robotizada.

\section{ABSTRACT}

According to Antonie Picon in "Ornament: the politics of architecture and subjectivity", the return of ornamentation as a practice today has several continuities with the classical ornament in terms of the subjectivity and politics.

In this context, the first objective of this article is to revise the concept of subjectivity in contemporary ornament/ architecture considering the ideas developed by the "object-oriented ontology" theory. Taking for instance an object created by a multi-agent system and manufactured with a clay extruder attached to a robotic arm, we wonder if the contemporary ornamental practice could continue to be referred as such, if the existence of the subject is no longer resolving and regulating its design and execution.

Secondly, the idea is to speculate if designers, in the context of post-work and full automation theories, will rediscover the sense of architectural forms that might give a political meaning to the ornament today.

Key words: Ornament, decoration, architecture, post-humanism, post-work, agent-base programming, objectoriented ontology, object-oriented programming, digital fabrication, robotics. 


\section{INTRODUCCIÓN}

"Humans are no longer monarchs of being, but are instead among beings, entangled in beings, and implicated in other beings."

LEVI R. Bryant. The Democracy of Objects

En el libro Inventing the Future: post-capitalism and a world without work, Nick Srnicek y Alex Williams proponen un programa político/cultural para desarrollar una sociedad post-trabajo. Considerando la evolución de los avances tecnológicos en términos de producción y sus posibles consecuencias en el sistema político actual, los autores proponen un nuevo orden basado en una economía plenamente automatizada, el achicamiento de la semana laboral, la implementación de un ingreso básico universal (UBI) y el desarrollo de una nueva cultura de trabajo.

Por otro lado, en su libro What is post-humanism? Cary Wolfe define el post-humaninsmo como la coyuntura histórica que rechaza la división humanista clásica entre cuerpo y alma, sociedad y naturaleza, humano y animal, orgánico y técnico, y redefine lo humano y sus características por su imbricación con redes técnicas, medicas, informáticas y económicas. En un sentido similar, en Alien Phenomenology lan Bogost define la "object-oriented ontology" (ontología orientada a los objetos) como la filosofía que ubica a las cosas en el centro de la existencia, al hombre como un elemento más de esa agregación y descarta la idea de jerarquía dentro de este sistema de relaciones por la de una "ontología plana” (Levi Bryant).

Por último y girando completamente de tema, en Ornament: the politics of architecture and subjectivity, Antoine Picon propone la reinvención del ornamento y el retorno de la ornamentación como práctica en la arquitectura contemporánea. A través de sus páginas, define la dimensión política y subjetiva del ornamento clásico, especula acerca de la nueva subjetividad y argumenta la falta de un sentido y significado político en las prácticas de hoy.

En este contexto, el objetivo de este artículo es reflexionar acerca de la dimensión subjetiva y política del ornamento arquitectónico en las practicas contemporáneas, valiéndonos de algunas ideas sobre post-humanismo y postrabajo definidas en los textos de Wolfe, Bogost, Srnicek y William respectivamente. Con el fin de reflexionar acerca del estado actual de la arquitectura, y no de profundizar conceptos filosóficos, nos serviremos del trabajo, la bibliografía y las ideas llevadas a cabo en los estudios de Matías del Campo y Sandra Ninninger, y Robert Stuart-Smith en la Escuela de Diseño de la Universidad de Pennsylvania.

\section{ORNAMENTO ORIENTADO A LOS OBJETOS}

A comienzos de siglo XXI, diseñadores e historiadores de la arquitectura hacían referencia a través de sus prácticas, libros, exhibiciones 


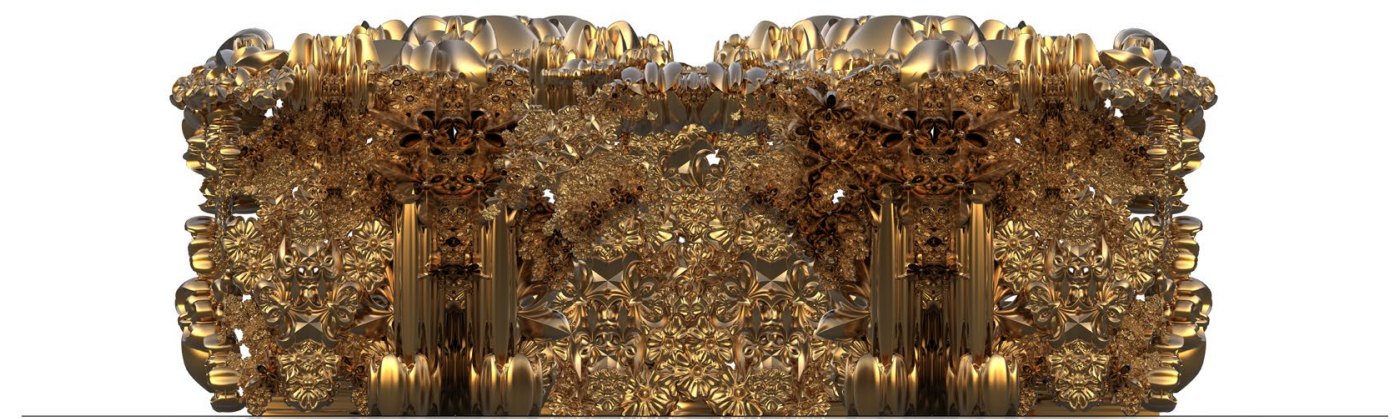

Figura 1.

Gilded Playhouse. Render de la envolvente exterior.

y conferencias al retorno del ornamento en la teoría y arquitectura contemporánea. Dentro de ese universo, Antoine Picon, profesor de Historia de la Arquitectura y Tecnología de la Escuela de Graduados de Diseño de la Universidad de Harvard, hizo una invaluable contribución con su libro Ornament: the politics of architecture and subjectivity.

Lo que nos interesa aquí del argumento de Picon es, en principio, la idea de que el ornamento ha vuelto, pero su "retorno" difiere de las practicas pasadas. En este sentido, mientras que el ornamento clásico era desmontable, limitado, puntual y distinto según se ubicaba hacia el exterior o interior de un edificio, el ornamento contemporáneo se caracteriza por ser repetitivo, completo, gigante, superficial, táctil y homogéneo (Figura 1). A pesar de las diferencias, Picon argumenta que existen elementos de continuidad entre el ornamento clásico y su reinvención contemporánea. Estos elementos se organizan según dos hilos conductores: el primero abarca la pregunta por la subjetividad y el segundo, la política.

Ahora bien, ¿de qué se trata esa dimensión subjetiva de que la habla Picon?, o en palabras del mismo autor ¿por quién y para quién se ornamentaba? Tradicionalmente, la subjetividad del ornamento estaba el diseñador que lo creaba, el artesano que lo producía y el destinatario para quien el objeto era construido. El ornamento reflejaba la autoridad del diseñador, su genio, imaginación y personalidad, la habilidad y destreza manual 
del trabajador y el decor, la jerarquía y los valores sociales de quien lo recibía. En este orden de cosas, la división entre el ornamento y el sujeto era notoria.

Actualmente, el subjeto se relaciona con el ornamento por su capacidad de afectarse mutuamente dentro de un mismo ambiente con él. En este sentido, argumenta Picon, se puede hablar de una "enviromental subjetivity": un sujeto observador, inmerso en un ornamento gigante, superficial y organizado según patrones fácilmente comprensible por él. La paradoja de este nuevo sujeto es que ya no puede ser reducido a un individuo permanentemente: más bien es individuo y ambiente al mismo tiempo. Además de la paradoja existe una ambigüedad posible de definir con la siguiente pregunta: ¿es quien diseña, produce y experimenta el ornamento el mismo sujeto? $\mathrm{Y}$ en esta ambigüedad nos gustaría detenernos para preguntarnos: ¿cuál es el rol de este diseñador/ productor en la práctica contemporánea?, ¿cómo se define su accionar frente a la existencia de técnicas y herramientas de diseño y fabricación digital?, ¿puede ser que la dimensión subjetiva del ornamento contemporáneo haya sido desplazada por una relación "object-oriented"?

En primer lugar, recordemos que según lan Bogost la posición "object-oriented" abandona la idea de que "el sujeto se posiciona en el centro de la existencia, organizando y regulando su devenir como si fuera un relojero" ${ }^{1}$. En este sentido las cosas no se relacionan meramente a través de los seres humanos, sino a través de cualquier uso, incluyendo la relación de un objeto con otro. En este orden de cosas, así como nada puede ser reducible por la existencia humana, tampoco lo es frente a otras cosas $^{2}$. Si se tradujeran estas ideas a la práctica de la arquitectura hoy, el diseñador ya no ocuparía una posición central en la práctica, sino que pasaría a ser un agente en relación con otros objetos (programas, códigos, simulaciones, robots) con capacidad de influenciar y ser influenciado por ellos. Para desarrollar estas ideas traeremos un ejemplo: el diseño de un objeto-columna utilizando un sistema de multiagentes y su impresión en tres dimensiones con un extrusor de arcilla adjunto a un brazo robótico. Este objeto fue diseñado en el marco de un seminario dictado en la Universidad de Pennsylvania por el arquitecto Robert StuartSmith.

En el proceso de formación del objeto-columna intervienen múltiples individuos: por un lado, el programador, el sistema y sus agentes, el ejecutor del sistema, el material, el brazo robótico, entre otros elementos (Figura 02). E programador se encarga de crear o diseñar el sistema compuesto por agentes, un entorno y

\footnotetext{
1 "one must abandon the belief that human access sits at the center of being, organizing and regulating it like an ontological watchmaker" (BOGOST, 2012, 5)

2 "all things equally exist, yet they do not exist equally" (BOGOST, 2012, 11)
} 


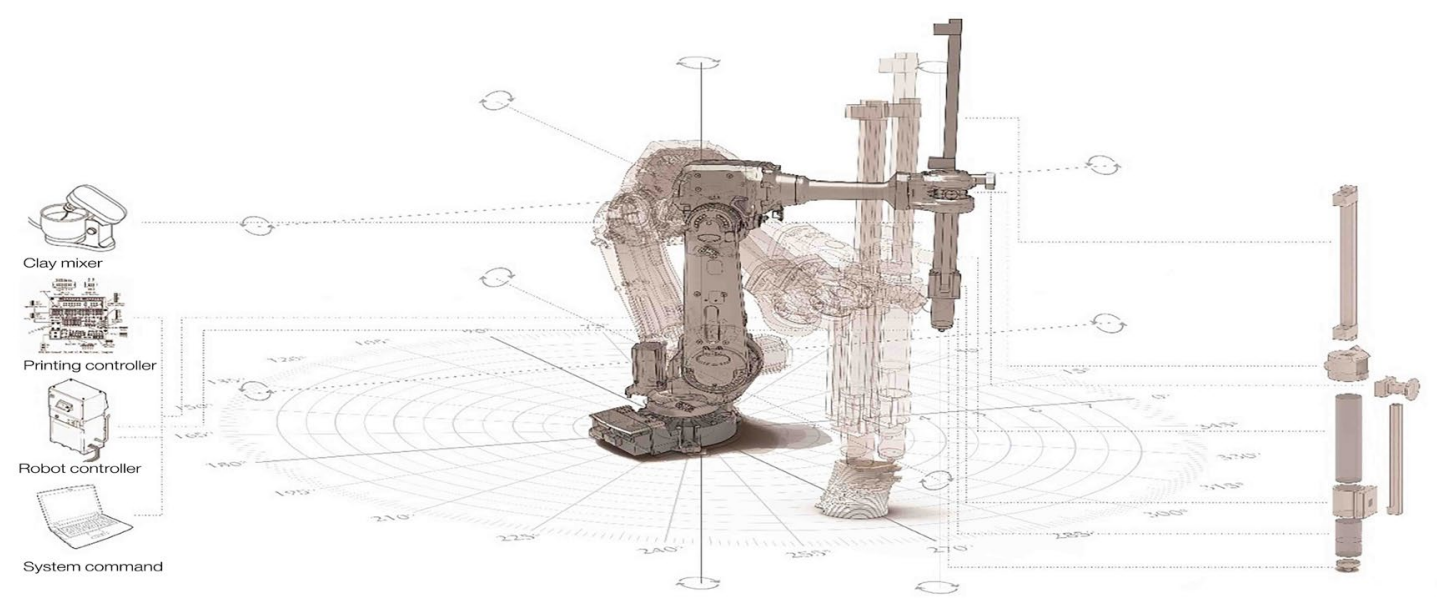

Figura 2.

Relaciones object-oriented. Agentes que intervienen en la producción del objeto-columna.

parámetros que condicionan su interacción. Los agentes son entidades individuales, dotadas de autonomía, que han sido programadas con capacidad para la toma de decisiones. En el caso de nuestro objeto, cada línea de contorno fue definida por el accionar de un agente que determinó el patrón que se observa sobre la superficie (Figura 03). Las formas que ofrece el programa son tantas como variaciones de los parámetros del sistema y sus posibles combinaciones. Es aquí donde el programador o diseñador decide, antes de correr el programa, los valores de cada parámetro, programados previamente por él, pero cuyos resultados son aleatorios según el comportamiento de los agentes. Es decir, un parámetro puede significar muy poco de manera aislada, pero puede ser determinante para cierta forma según el valor del resto de los parámetros (Figura 04a-b).
Algunos de los parámetros usados en este caso son velocidad de los agentes, ángulos de trayectoria, escala, tiempo de recorrido, etcétera.

Una vez obtenidos los resultados deseados, se debe programar el código que leerá el brazo robótico y replicará la trayectoria de los agentes. Recordemos que nuestro brazo robótico sostenía un extrusor de arcilla, así que no solo replicará la trayectoria de los agentes, sino que también depositará material a lo largo de su recorrido, según una velocidad previamente programada. El resultado dependerá no solo del diseño del programa y la velocidad de movimiento del brazo, sino también de la mezcla, consistencia y calidad de la arcilla, y la técnica de rellenado del extrusor (Figura 05). 

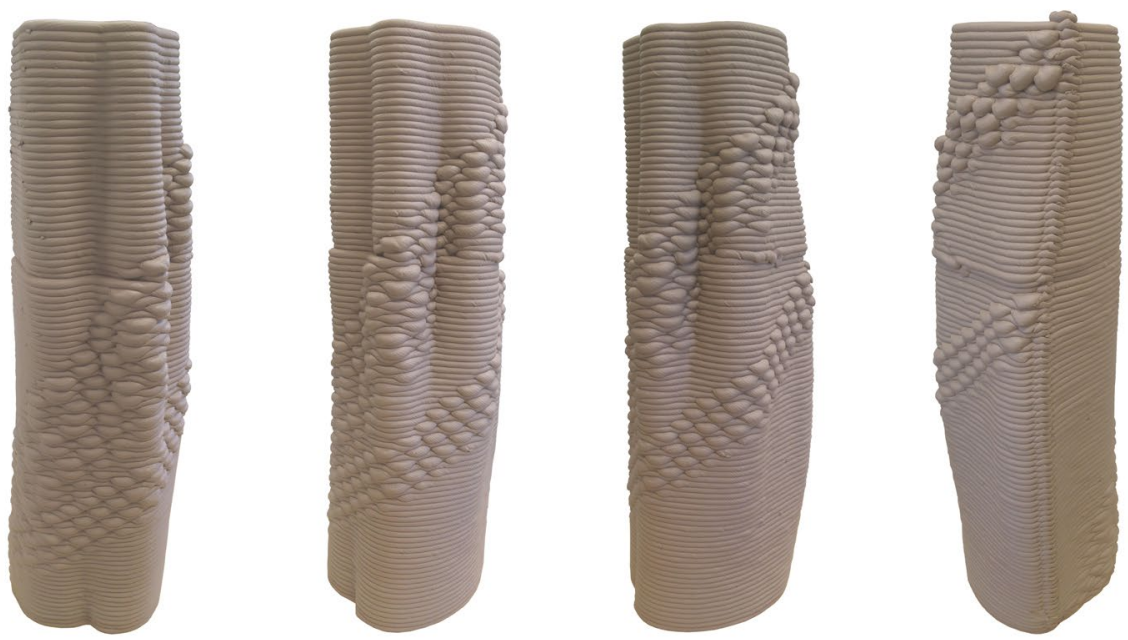

Figura 3.

Objeto-columna diseñado con un sistema de multi-agentes programado en Phyton para Rhino e impreso en arcilla.

El objeto resultante de este proceso es una superficie con cualidad ornamental. El sujeto programador, diseñador y fabricante, el sistema de multi-agentes, la arcilla, el brazo robótico intervinieron en su proceso de formación, (brevemente detallado en el párrafo anterior) de forma cooperativa pero autónoma. Es decir, en este universo de producción, sujetos y objetos trabajaron en un colectivo común pero su existencia o significado, dentro del proceso, no puede limitarse exclusivamente a la existencia o significado de otro: en palabras de Bogost “los significados de una unidad para dar sentido a otra no son universales y no pueden explicarse a través de la ley natural, la verdad científica o incluso su propia perspectiva"3. En este sentido nos volvemos a preguntar: ¿es posible que la dimensión subjetiva del ornamento contemporáneo haya sido desplazada por una relación "object-oriented" ? Si desaparece el "hilo" de la subjetividad que vinculaba, según Picon, el ornamento clásico con el contemporáneo, ¿de qué se trata esta práctica tan común en la arquitectura de hoy?, ¿qué sucedería si el ornamento contemporáneo, al perder subjetividad, fuera en última instancia simplemente arquitectura?

3 "A unit's means of making sense of another is not universal and cannot be explained away through natural law, scientific truth, or even its own perspective". (BOGOST, 2012, 30) 

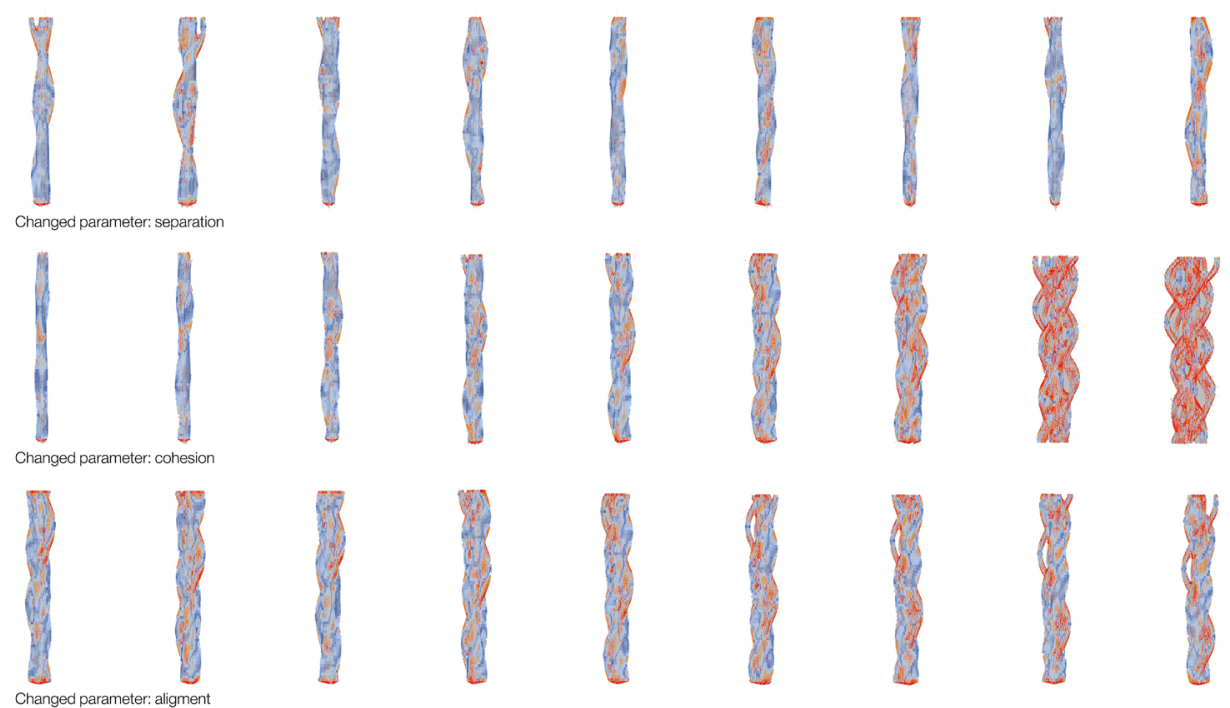

Physics-based sir Optimizationof themesh stability

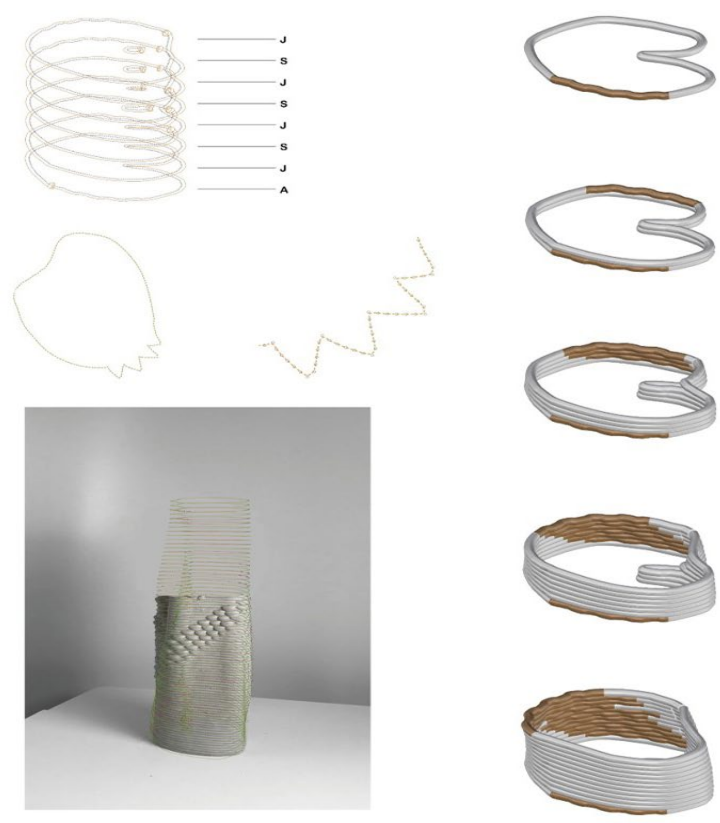

Figura 4 a-b.

Construcción del objeto con sistema de multi-agentes. 


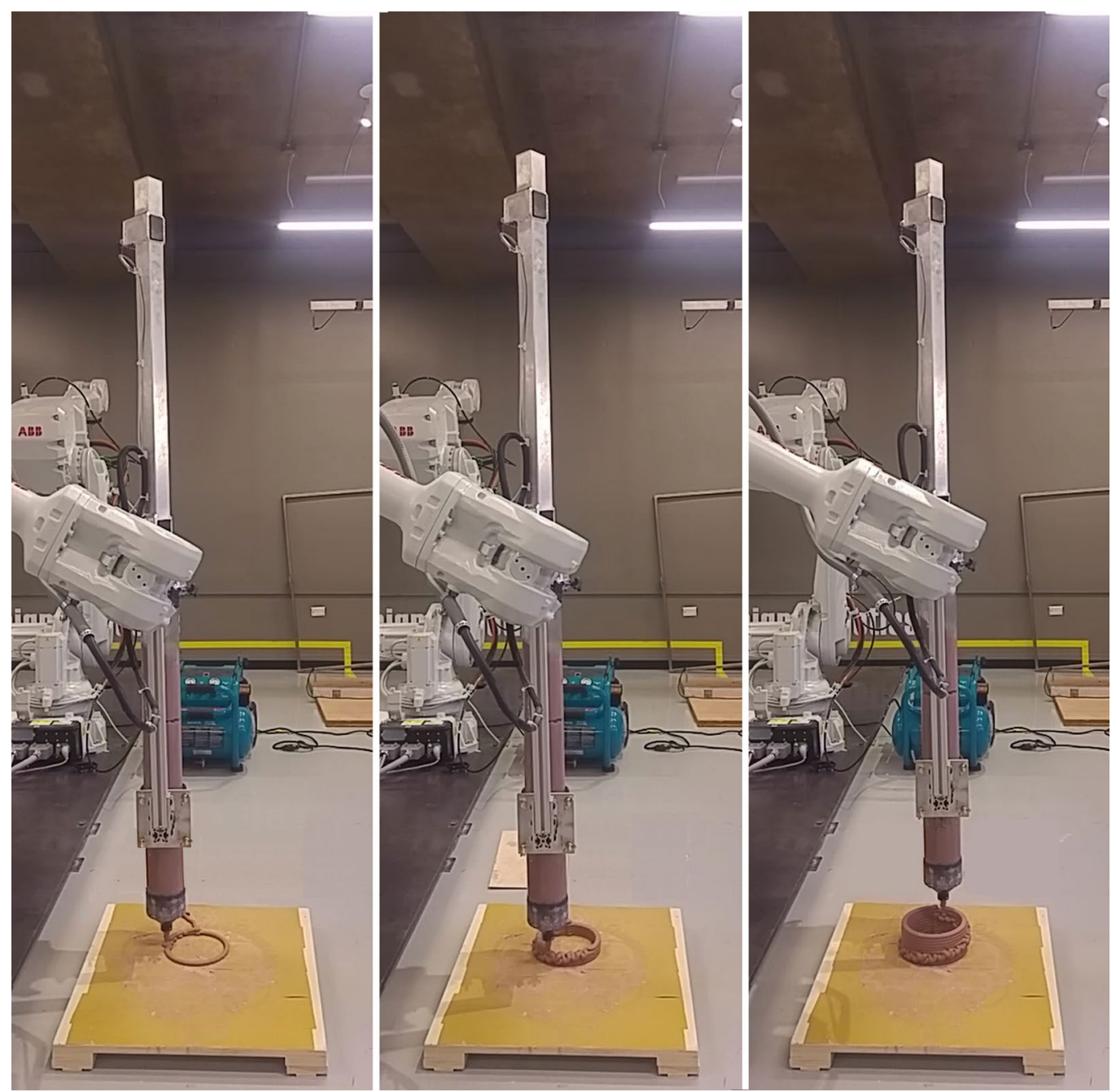

Figura 5.

Impresion 3d con un extrusor de arcilla. 


\section{ORNAMENTO TOTALMENTE AUTOMATIZADO}

"liberate humanity from the drudgery of work, the dependence on wage labour, and the submission of our lives to a boss"

SRNICEK, Nick. Inventing the Future: Postcapitalism and a World Without Work

Hasta aquí hemos trabajado la dimensión subjetiva que, según Picon, une el ornamento clásico con su reinvención contemporánea. Queda, sin embargo, una segunda dimensión: la pregunta por el sentido político en el ornamento de hoy.

Tradicionalmente, este sentido o significado político estaba vinculado con el diseñador, el productor y el dueño o destinatario del ornamento. El objeto estaba llamado a "revelar el ser" de este destinatario: debía decir algo sobre él y por lo tanto era fabricado específicamente por y para él. El ornamento era comunicación: no solo representaba la prosperidad económica y el poder de su portador (ya sea individuo o institución), sino también el genio del diseñador, y la destreza y técnica del artesano que lo creaban. Las relaciones entre el diseñador, el artesano y el dueño del objeto generaban una serie de negociaciones que dotaban, nuevamente, de un sentido político al ornamento.

Actualmente, el artesano y el diseñador tienden a ser, como consecuencia de la incorporación de técnicas de fabricación digital, un mismo agente en un sistema de producción que interactúa o negocia con otros objetos (recordemos las ideas de lan Bogost sobre la posición “object-oriented). Las limitaciones de la técnica, antes impuestas por las herramientas y la destreza del artesano, ahora aparecen, por ejemplo, en el diseño de efectores finales para brazos robóticos. En este sentido, si observamos las técnicas de corte robóticos de bloques de poliestireno con cable caliente aparecen dos claras limitaciones: en primer lugar, nunca podríamos cortar el bloque en el sentido perpendicular al efector ya que lo quemaríamos (suponiendo que el bloque esta soportado por el brazo) y, en segunda instancia, con un cable tensado, nunca podríamos conseguir otro tipo de superficie que no sea reglada (Figura 06). Si bien estos problemas pueden resolverse modificando la posición del cable y del bloque, lo que queremos ilustrar es que las antiguas negociaciones y tensiones entre el diseñador y el artesano, que dotaban de sentido político al ornamento, fueron reemplazadas por relaciones entre el diseñado/ fabricante y un nuevo repertorio de objetos que lo rodean. Asimismo, estos nuevos objetos de fabricación generan negociaciones y tensiones que se traducen al espacio de trabajo, en donde cada objeto está limitado al uso de su espacio individual para no entrar en conflicto con otros miembros del sistema de producción. 

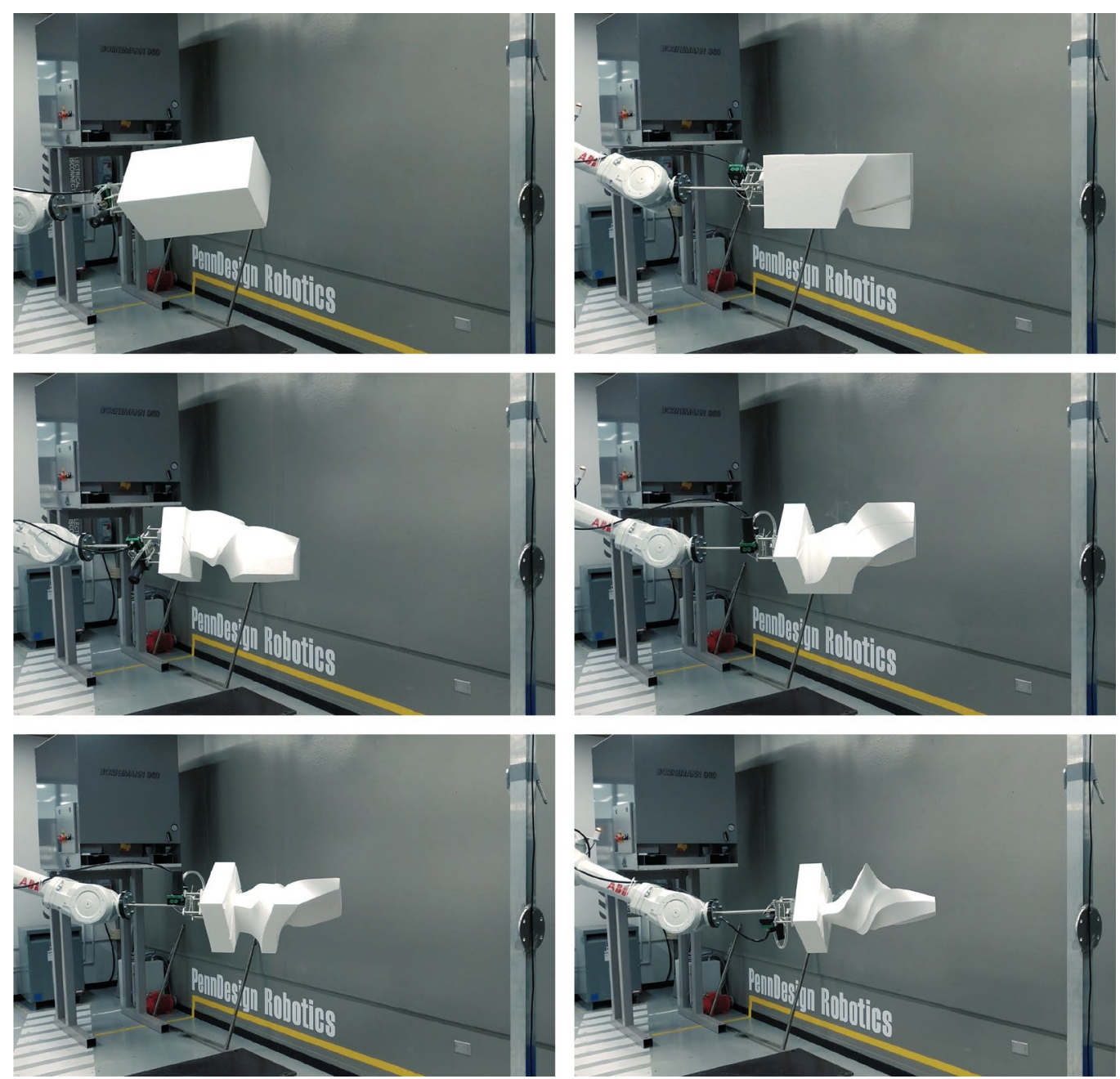

Figura 6.

Corte robóticos de bloques de poliestireno con cable caliente. 
Sin embargo, la pregunta por la dimensión política parece aun inconclusa a pesar de la existencia de las negociaciones. Según Picon, el ornamento tenía un sentido político que comunicaba a través de significados y símbolos. Hoy las ideas de comunicación y significado en arquitectura son, según él, un tabú. Ahora bien, si el significado era capaz de relacionar la arquitectura, a través de su ornamentación, con la sociedad, ¿es posible que un cambio de orden social, como la cultura de post-trabajo, transforme la relación arquitectura-sociedad y por lo tanto dote al ornamento de un nuevo significado político?

Si bien Picon argumenta que la dimensión política del ornamento no puede resolverse al nivel de programas, ideologías o partidos políticos circunstanciales ${ }^{4}$, nuestro objetivo es especular acerca de un escenario en donde la hegemonía del trabajo dependiente y asalariado se vea desplazado, como sostiene Nick Srnicek y Alex Williams, por otros tipos de trabajos y esfuerzos que comúnmente asociamos con el esparcimiento y el placer, pero que muy lejos están de ser ociosos: leer un libro, tocar un instrumento, socializar, etcétera . Si la cultura del post-trabajo propone la plena automatización del trabajo, y por lo tanto el balance entre cantidad de trabajo dependiente

4 "Of course, the problem does not lie at the level of political parties and programs. One should not reduce the relation between architecture and politics to the ideological role that it has played at certain moments in history, albeit not always successfully" (PICON, 2013, 145) y placentero, con el objetivo de promover este último y en consecuencia dotar a las personas de mayor tiempo para realizar actividades culturales, ¿podría ocurrir una opulencia y abundancia de producción cultural? Si el ornamento está tradicionalmente asociado al placer y a la belleza, ¿es posible que este nuevo orden social y cultura del trabajo incentive la practica ornamental y su sentido político de una forma no concebida hasta el momento?

En el marco de la plena automatización de la producción creemos que distintas prácticas formales pueden tener lugar. Por un lado, esta realidad habilitaría la creación de objetos arquitectónicos con una cualidad y en una cantidad antes impensada: la producción automatizada permitiría elaborar objetos customizados con alto nivel de precisión en poco tiempo y las personas tendrían más lugar para reflexionar, producir y asignar significados a las nuevas creaciones. Por otro lado, y retomando las ideas del post-humanismo, creemos que esta nueva producción cultural se vería formalmente afectada por el hecho de ser producida por un arquitecto/fabricante embebido en relaciones "democráticas" con otros objetos que producen a la par de él. En este contexto, especulamos que la nueva producción cultural ya no se organizaría en función de las dicotomías ontológicas conocidas del humanismo, sino que daría lugar a nuevas formas producto de diversas naturalezas en donde los límites, por ejemplo, entre infraestructura y naturaleza, estructura y 

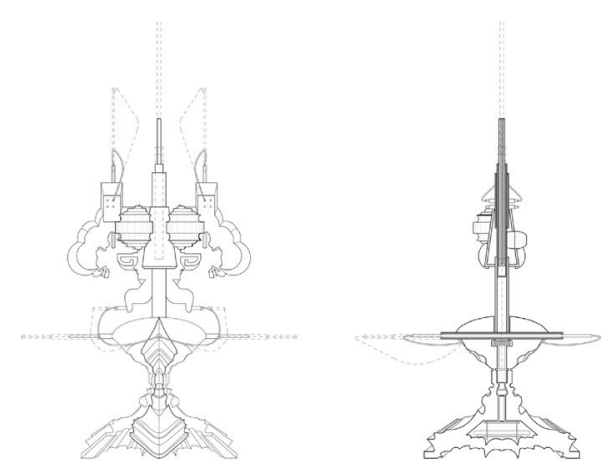

Figura 7.

"Decorated machinery objects".

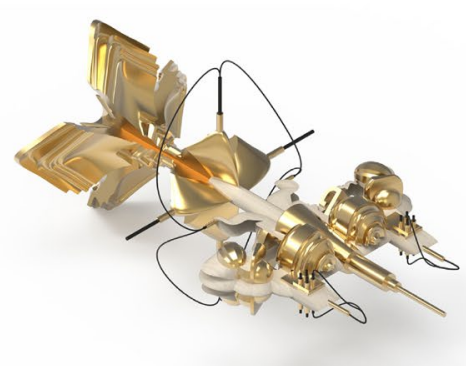

Decorated-machinery object-Auditory
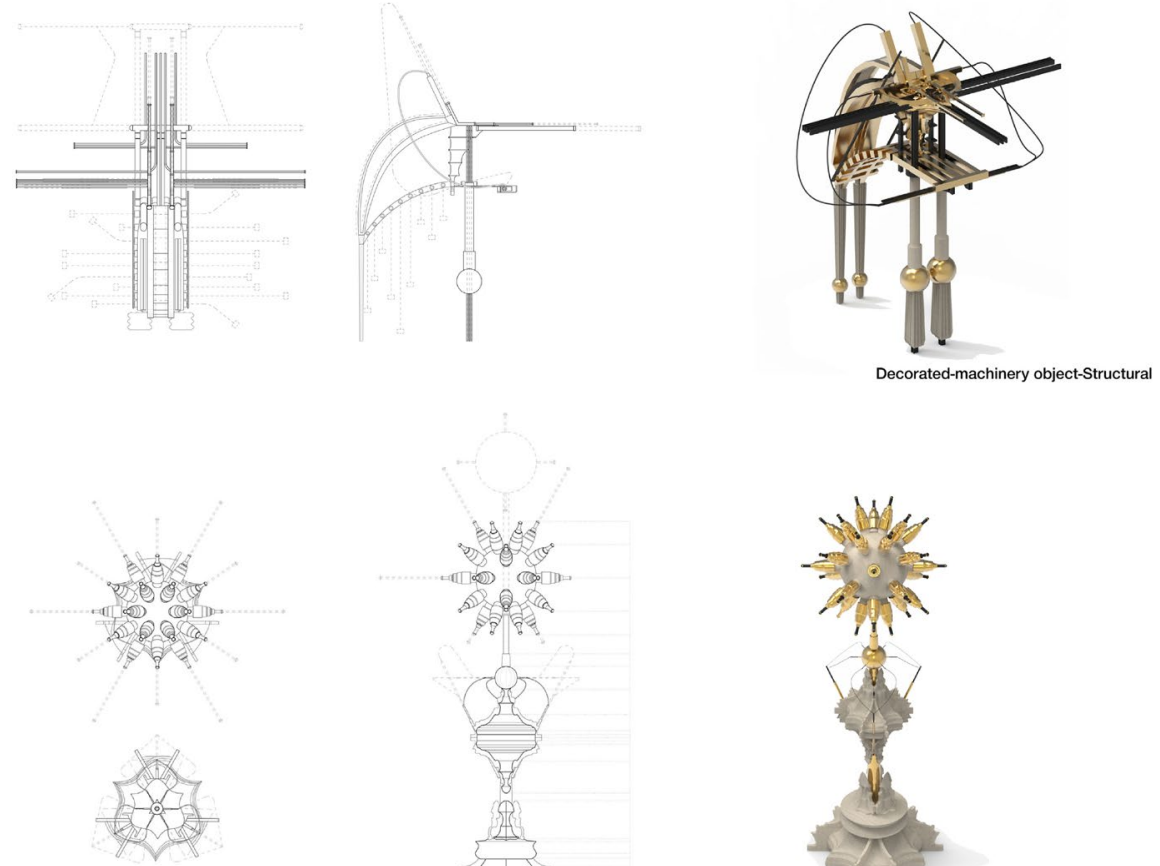

Decorated-machinery object-Visual
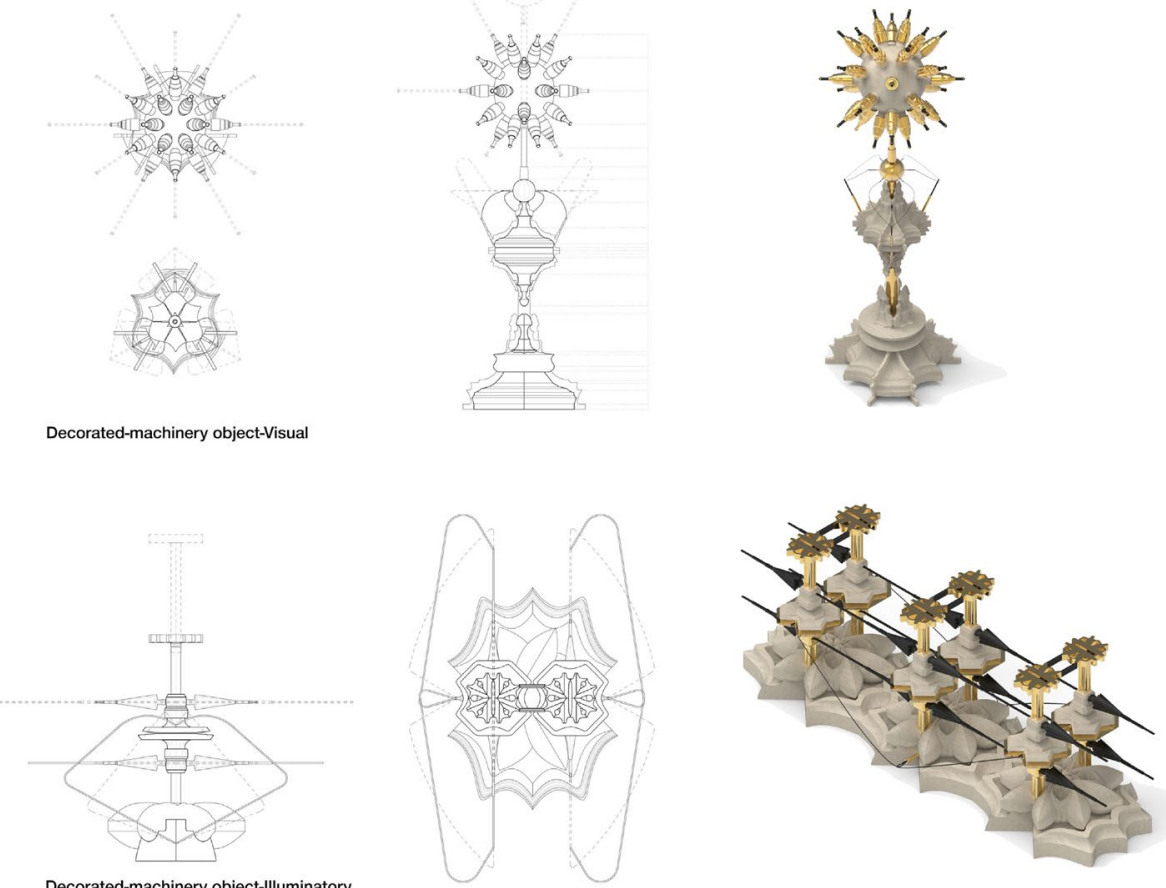

78 Anales de Investigación en Arquitectura Vol.9 | 2019 | № 1. Montevideo (Uruguay), 65-82 Universidad ORT Uruguay 
ornamento, viejo y nuevo, estarían suavizados y remodelados con el fin de construir una nueva realidad hibrida, múltiple y compleja.

Con el fin de ilustrar esta descripción nos serviremos del diseño de cuatro objetos, pensados para el interior de un edificio "soundstage" de una productora de cine, hechos en el estudio de Matías del Campo y Sandra Ninninger en la Universidad de Pennsylvania (Figura 7). El objetivo de estas piezas era producir una serie de objetos híbridos entre formas ornamentales clásicas y objetos del universo cinematográfico como cámaras $360^{\circ}$, luces, micrófonos y andamios. Asimismo, las piezas estaban diseñadas para operar de manera autónoma e incluían fragmentos del repertorio formal de los objetos automatizados como cables, mangueras, enchufes, etcétera. Así, la idea era formar objetos híbridos en donde los límites entre lo antiguo y lo nuevo, lo ornamental y lo maquínico, lo estructural y lo móvil, se vieran borrados en favor de un objeto múltiple, variado y complejo.

En este sentido y para finalizar nos preguntamos: si la sociedad de post-trabajo y el advenimiento de la completa automatización de la producción incentivan el diseño y la producción cultural, de la cual el ornamento forma parte, de una forma no concebida hasta aquí ¿es posible que no solo la arquitectura se vuelva ornamental, sino que la sociedad per se y todas sus creaciones tiendan a convertirse en ornamento?

\section{CONCLUSIÓN}

A principio de nuestro articulo revisamos la dimensión subjetiva del ornamento definida por A. Picon en su libro Ornament: the politics of architecture and subjectivity. Picon sostiene que la subjetividad es el primero de dos hilos de conexión que vinculan el ornamento clásico con su reinvención contemporánea. Ahora bien, nuestro argumento sostiene que la subjetividad clásica del ornamento (el diseñador, el artesano y el portador del objeto) han sido desplazados, en la práctica contemporánea, por relaciones "object-oriented". En este sentido, las figuras tradicionales del diseñador y artesano que ocupaban una posición central, jerárquica y reguladora del hacer son, en las prácticas de hoy, un agente en relación con una serie de objetos (programas, brazos robóticos) con ontología propia. Valiéndonos de un objeto ornamental impreso en $3 d$ con un extrusor de arcilla adjunto a un brazo robótico y diseñado con un sistema de multi-agentes, ejemplificamos el sistema de relaciones entre el diseñador-fabricante, el programa, el brazo robótico, el material, en donde las influencias ya no son unilaterales por parte del diseñador, sino múltiples y reciprocas entre objetos.

En relación a la dimensión política del ornamento consideramos el texto de Nick Srnicek y Alex Williams, Inventing the Future para especular acerca de un posible escenario en donde el ornamento cobre sentido debido a una transformación de la sociedad 


\section{BIBLIOGRAFÍA}

y su cultura del trabajo: si la producción se automatiza completamente, las personas se verían liberadas del trabajo dependiente a favor de otras actividades culturales y labores placenteros en donde el diseño y producción del ornamento tendrían lugar.

En este sentido y para concluir, nos repreguntamos si al desplazar la subjetividad y asignarle un nuevo sentido político del ornamento contemporáneo este puede seguir definiéndose como entidad individual o es en, última instancia, de lo que se trata la arquitectura y las creaciones de las personas en el universo de hoy.
BOGOST, I. (2012). Alien phenomenology, or, What it's like to be a thing. Minneapolis: University of Minnesota Press.

BRYANT, L. R (2011). The Democracy of Objects. Open Humanities Press Imprint.

PICON, A. (2013). Ornament: The politics of architecture and subjectivity. John Wiley \& Sons, Incorporated, New York.

PAWAR, VIJAY, STUART-SMITH, ROBERT, \& SCULLY, PETER. (2017). Toward autonomous architecture: The convergence of digital design, robotics, and the built environment. Science Robotics, 2(5).

STUART-SMITH, R. (2016). Behavioural Production: Autonomous Swarm-Constructed Architecture. Architectural Design, 86(2), 54-59.

SRNICEK, N., \& WILLIAMS, A. (2015). Inventing the future: postcapitalism and a world without work. Verso Books Brooklyn, NY.

WERFEL, J., PETERSEN, K., \& NAGPAL, R. (2014). Designing collective behavior in a termiteinspired robot construction team. Science (New York, N.Y.), 343(6172), 754-8.

WOLFE, C. (2010). What is posthumanism? Minneapolis: University of Minnesota Press. 


\section{FUENTE DE LAS IMÁGENES}

Figura 1: University of Pennsylvania Stuart Weitzman School of Design - Tutores: Matías del Campo y Sandra Ninninger - Autores: Sarah E. Davis, Mariana Righi.

Figura 2: University of Pennsylvania Stuart Weitzman School of Design - Tutor: Robert Stuart-Smith - Autores: Mostafa Akbari, Mariana Righi, Cao Xinyue, Dai Wenna, He Yuting, Ma Yuan.

Figura 3: University of Pennsylvania Stuart Weitzman School of Design - Tutor: Robert Stuart-Smith - Autores: Mariana Righi, Mostafa Akbari, Cao Xinyue, Dai Wenna, He Yuting, Ma Yuan.

Figura 4 a-b: University of Pennsylvania Stuart Weitzman School of Design - Tutor: Robert Stuart-Smith - Autores: Mariana Righi, Mostafa Akbari, Cao Xinyue, Dai Wenna, He Yuting, Ma Yuan.

Figura 5: Autonomous Manufacturing Lab University of Pennsylvania Stuart Weitzman School of Design - Director: Robert StuartSmith - Investigadora: Mariana Righi.

Figura 6: Autonomous Manufacturing Lab - University of Pennsylvania School of Design. Director: Robert Stuart-Smith Investigadores: Musab Badahdah, Mariana Righi

Figura 7: Advanced Architectural Studio University of Pennsylvania Stuart Weitzman School of Design - Tutores: Matías del Campo y Sandra Ninninger - Autores: Sarah E. Davis, Mariana Righi. 
82 Anales de Investigación en Arquitectura Vol.9 | 2019 | № 1. Montevideo (Uruguay), 65-82 Universidad ORT Uruguay 